\title{
Sistema convectivo de mesoescala atuantes na região semiárida do nordeste do Brasil
}

\author{
Micejane da Silva Costa ${ }^{a}$, Kellen Carla Lima ${ }^{b}$
}

aDoutora em Ciências Climáticas pelo Programa de Pós-Graduação em Ciências Climáticas (PPGCC/UFRN); Pesquisadora no Laboratório de Meteorologia e Meio Ambiente (LAMMA/ICAT/UFAL), CEP 57.072-970, Maceió, AL, Brasil.

bProfessora Dra. da Escola de Ciência e Tecnologia e do Programa de Pós-Graduação em Ciências Climáticas, Universidade Federal do Rio Grande do Norte (PPGCC/UFRN), CEP 59.072-970, Natal, RN, Brasil.

RESUMO Nesse estudo, buscou-se realizar um levantamento bibliográfico a respeito dos Sistemas Convectivos de Mesoescala (SCM) que influenciam na ocorrencia de eventos extremos de precipitação na região Semiárida do Nordeste do Brasil (SANEB). Nesta revisão de literatura foram utilizados artigos científicos nacionais e internacionais, publicados nas plataformas Scielo, Elsevier e periódicos Mendeley. A motivação desta pesquisa deve-se à carência de informações relacionados ao tema escolhido, com base na preocupação de dar subsídios que favoreçam ao conhecimento acadêmico sobre os SCM. Para tanto, são apresentadas as definições, o período de atuação, sua origem, e seu ciclo de vida no qual inclui sua formação, desenvolvimento e dissipação. Este estudo faz-se necessário, visto que o conhecimento antecipado das condições dinâmicas da atmosfera favorece ao desenvolvimento e manutenção destes eventos, possibilitando caracterizar o ambiente sinótico dos mesmos.

PALAVRAS-CHAVE chuvoso; CCM; precipitação; SCM; semiárido

Aceito 15 de janeiro de 2020 Publicado online 08 de fevereiro de 2020

Cite este artigo: Costa MS, Lima KC (2020) Sistema convectivo de mesoescala atuantes na região semiárida do nordeste do Brasil. Multidisciplinary Reviews 3: e2020002 DOI: $10.29327 /$ multi. 2020002

\section{Mesoscale convective system acting in the semiarid region of Northeastern Brazil}

ABSTRACT In this study, we sought to conduct a bibliographic survey about the Mesoscale Convective Systems (MCS) that influence the occurrence of extreme precipitation events in the semiarid region of northeastern Brazil (NEBSA). In this literature review were used papers national and international published in Scielo platforms, Elsevier and periodicals Mendeley. The motivation of this research is due to the lack of information related to the chosen theme, based on the concern of giving subsidies that favor the academic knowledge about MCS. To this end, the definitions, the period of operation, their origin, and their life cycle in which they include their formation, development and dissipation are presented. This study is necessary, since the advance knowledge of the dynamic conditions of the atmosphere favors the development and maintenance of these events, making it possible to characterize their synoptic environment.

KEYWORDS: CMC, MCS, precipitation, rainy, semiarid

\section{Introdução}

A variável precipitação é considerada como a mais importante na região tropical, visto que está diretamente relacionada com a convecção local. Esta convecção é caracterizada por movimentos ascendentes de ar quente e úmido, resultantes da ocorrência de pressões atmosféricas mais baixas junto a superfície (Molion e Bernardo 2002). Os Sistemas Convectivos de Mesoescala (SCM) é um exemplo de sistema resultante dessa convecção. Esses SCM são sistemas que possuem curta duração, porem podem alcançar grandes dimensões (Maddox 1980; Costa et al 2019), favorecendo a eventos extremos de precipitação, por isso, a necessidade de maior conhecimento dos mesmos. Nesse sentido, ao se propor uma revisão bibliográfica para detalhar as características físicas e morfológicas dos SCM, favorece o 
conhecimento da comunidade em geral. Diante disso, o objetivo dessa revisão é realizar um levantamento bibliográfico a respeito dos SCM atuantes sobre o SANEB.

\section{Sistemas convectivos de mesoescala}

Segundo Orlanski (1975), os sistemas meteorológicos possuem diferentes tipos de escalas atmosféricas na qual inclui micro, meso, sinótica e grande escala, como também, a escala sinótica. Porém, neste artigo foram abordados os Sistemas Convectivos de Mesoescala (SCM) que influenciaram e influenciam na precipitação da região SANEB, compreendidos na escala do tipo meso (Tabela 1). Na mesoescala observam-se os SCM que podem ser subdividos em Complexos Convectivos de Mesoescala (CCM), Linhas de Instabilidade (LI) e o Sistema Frontal (SF), que variam entre 20 e $2500 \mathrm{~km}$, com duração acima de $6 \mathrm{~h}$ podendo chegar a 7 dias.

Tabela 1 Escala do SCM conforme Orlanski (1975).

\begin{tabular}{|c|c|c|c|c|}
\hline Tipo & Divisão & Dimensão horizontal & Duração & Fenômenos \\
\hline & Meso- $\gamma$ & $2-20 \mathrm{~km}$ & $1 \mathrm{~h}$ & $\begin{array}{l}\text { Tempestades locais, Turbulência } \\
\text { de céu claro. }\end{array}$ \\
\hline \multirow[t]{2}{*}{ Mesoescala } & Meso- $\beta$ & $20-200 \mathrm{~km}$ & $1 \mathrm{~h}$ a 1 dia & $\begin{array}{l}\text { Jato de baixos níveis (JBN), } \\
\text { Tempestades locais, circulações } \\
\text { locais, LI. }\end{array}$ \\
\hline & Meso- $\alpha$ & $200-2.500 \mathrm{~km}$ & 1 a 7 dias & $\begin{array}{l}\text { SCM, LI, CCM, Correntes de Jatos, } \\
\text { pequenos Furacões e SF. }\end{array}$ \\
\hline
\end{tabular}

A denominação Sistemas Convectivos de Mesoescala (SCM) é dada ao conjunto de sistemas de aglomerados de nuvens convectivas, principalmente do tipo Cumulonimbus ( $\mathrm{Cb}$ ), observadas em diferentes estágios de ciclo de vida. Foram definidos por Maddox (1980) e confirmados por Houze (1993), como sistemas que se formam em tempestades individuais ou em formato de linhas.

\section{Local e período de atuação}

Devido suas formas variadas, foi possível classificar em Linhas de instabilidade (LI) os sistemas que possuem formatos de linha e em Complexos Convectivos de Mesoescala (CCM), os que apresentam um formato circular, ou simplesmente, em SCM, os de formas irregulares. Entretanto, esses sistemas possuem características em comum, tais como: precipitação convectiva e estratiforme, com tempo de vida de 6 a 12h, podendo durar vários dias (Houze 1977; Maddox 1980; Mcanelly e Cotton 1989; Houze 1993 e 2004; Eichholz 2011; Eichholz e Campos 2014; Costa et al 2019; Song et al 2019). De acordo com Orlanski (1975), os SCM apresentam escalas horizontais da ordem de $20-200 \mathrm{~km}$ (meso- $\beta$ ) e de 200 - 2.500 km (meso- $\alpha$ ), conforme observado na Tabela 1, e são responsáveis pela maior parte da precipitação nos trópicos e em várias localidades de latitudes médias durante a estação quente.

Barbosa e Correia (2005) investigaram a distribuição dos sistemas convectivos responsáveis por eventos extremos de precipitação e inundações no Rio São Francisco no ano de 1985. As autoras estudaram um evento incomum de atividade convectiva intensa no mês de junho. Seus resultados mostraram que o ambiente sinótico foi determinante na evolução dos SCM e que a convergência do fluxo de umidade na baixa troposfera foi fundamental para a ocorrência da convecção profunda.

Um dos primeiros estudos sobre SCM no semiárido do Brasil foi realizado por Ramos (1975). Machado e Rossow (1993) e Salio et al (2007) observaram uma ligação direta entre o tamanho médio do SCM e o seu tempo de vida. Os autores analisaram os SCM e sua relação com o jato de baixos níveis (JBN) no período de setembro de 2000 a maio de 2003, nas coordenadas $10^{\circ}$ a $40^{\circ} \mathrm{S}$ e $40^{\circ}$ a $75^{\circ} \mathrm{W}$ sobre a AS, e concluíram que, os SCM tropicais são menores e duram 
menos do que os SCM subtropicais. O que concorda com o estudo de Eichholz (2011) e que este, justificou que isso ocorre principalmente porque os SCM tropicais recebem calor e umidade devido ao aquecimento diurno, enquanto que os SCM subtropicais recebem calor e umidade trazidos pelo JBN.

\section{Ciclo de vida}

Formação

A formação dos SCM deve-se à união de células novas com células mais antigas, permitindo um composto multicelular de nuvens em questão de horas. Frequentemente, a evolução desses sistemas está associada à convecção profunda, cujo desenvolvimento ocorre normalmente devido a algumas forçantes que acionam a convecção. Esta convecção é ativada pela convergência de ar quente e úmido em níveis mais baixos da troposfera, desenvolvendo circulações de mesoescala à medida que amadurecem. Formam-se quando as nuvens que ocorrem em resposta à instabilidade convectiva, se organizam em um único sistema de nuvem de grande porte, abrangendo grandes áreas nos trópicos e nas latitudes médias, favorecendo a precipitação (Houze 1977; Maddox 1980; Houze et al 1990; Houze 2004; Eichholz 2011). O sistema apresenta-se como grupo de células isoladas, distribuídas de forma aleatória e alinhadas.

\section{Desenvolvimento}

No estágio de desenvolvimento, as células isoladas crescem e se fundem criando regiões de intensa precipitação interligadas por regiões de menor precipitação. A evolução desse sistema geralmente associa-se à convecção profunda, cujo desenvolvimento ocorre normalmente, devido ao aquecimento em baixos níveis, umedecimento na camada limite ou movimento ascendente em grande escala (Houze 2004). Uma das características importantes para se entender a evolução de um SCM é o comportamento do seu perfil vertical em determinadas variáveis meteorológicas como por exemplo, temperatura e umidade do ar. Segundo alguns autores (Maddox 1983; Cotton et al 1989; Guedes e Machado 1994; Laing e Fritsch 2000; Torres 2003; Pinto e Campos 2007) ao longo do ciclo de vida, o geopotencial, a temperatura, a umidade e a velocidade vertical evoluem de forma combinada entre as fases de iniciação, intensificação e dissipação. Seu desenvolvimento geralmente é semelhante em ambos Hemisférios, Norte e Sul.

\section{Dissipação}

Quando atinge seu desenvolvimento máximo, observa-se precipitação do tipo estratiforme na retaguarda do SCM, que se mantém enquanto novas células se formam na parte dianteira do sistema. Portanto, ocorre quando o SCM deixa de formar novas células e entra em fase de dissipação, isto porque a formação de novas células diminui e o mesmo não tem como se sustentar, provocando seu enfraquecimento (Houze 1993; Machado e Rossow 1993).

Existem dois tipos especiais de SCM que assumem organização espacial: Linhas de Instabilidade (LI) e Complexos Convectivos de Mesoescala (CCM), os quais são descritos na sequência.

\section{Linha de Instabilidade}

As Linhas de Instabilidade (LI) (Figura 2.1), são linhas de nebulosidade associada à convecção profunda, podendo ser contínua ou não (Cavalcanti 1982). As LI se dividem em duas categorias: (i) Tropicais formam-se ao longo da costa N-NE do Brasil como resultado da convecção pela brisa marítima associada às ondas de leste. Ocorrem nos meses de verão no período da tarde e início da noite. A proximidade da ZCIT e a fonte de calor sobre a Amazônia são outros fatores que contribuem para a formação e intensificação da LI tropicais. (ii) Latitudes Médias formam-se em latitudes médias e geralmente estão associadas à circulação pré-frontal, podendo aparecer tanto no setor quente quanto no setor frio e podem ser paralelas ou perpendiculares à frente. A umidade vinda do Oceano Atlântico associada à brisa marítima, juntamente com a convergência em baixos níveis, são favoráveis à formação e intensificação das LI (Garstang et al 1994; Cohen et al 1995). 
Ocorrem na costa norte-nordeste da América do Sul (AS), como também, nas latitudes médias. São observadas frequentemente, e podem se propagar para o interior do continente. As autoras Cavalcanti (1982) e Cohen (1989), sugeriram que a convecção nas LI está associada à brisa marítima, na costa atlântica norte, com deslocamento continente adentro quando o escoamento médio em $850 \mathrm{hPa}$ é perpendicular à costa, no entanto, a LI não se propaga quando o escoamento é paralelo à costa.

O primeiro a estudar as LI na região Nordeste do Brasil (NEB) foi Kousky (1980), o qual propôs que o aquecimento solar e a circulação de brisa seriam os principais mecanismos a considerar. Com início de formação no período entre o final da tarde e início da noite (Garstang et al 1994). Para classificar os tipos de LI, autores levaram em conta as LI que se formaram ao longo da costa norte-nordeste da América do Sul, desde a Guiana até o estado do Maranhão. Consideraram tanto as $\mathrm{LI}$ que se propagam e as que não se propagam, associadas à circulação de brisa marítima junto à costa. Segundo Cohen et al. (2009), o tempo médio de vida de uma LI é de 10 horas para as LIC e 20 horas para as LIP.

Cavalcanti (1982) realizou um estudo sobre a interação da LI com a ZCIT, e percebeu que, o local de formação da LI sofre um deslocamento latitudinal acompanhando a posição da ZCIT. Isto ocorre devido o deslocamento da ZCIT para norte, o que provoca movimentos subsidentes associados ao ramo descendente da célula de Hadley que atua sobre o NEB (Molion e Bernardo 2002) e inibe a formação de convecção na região de atuação.

Segundo Cohen et al (1995), 90\% da precipitação observada no norte do NEB, no período de 21 de abril a 3 de maio de 1987, estiveram associadas às LI que se propagaram para o interior do continente. O Distúrbio Ondulatório de Leste sobre o Atlântico Sul, observado no dia 5 de maio, foi um dos mecanismos a produzir intensificação no escoamento de leste, nos baixos níveis nos dias de atuação das LI. Observaram ainda, fontes tropicais de calor no oeste da Amazônia que parecem se intensificar de 4 a 6 de maio, o que causaria um aumento da convergência e, portanto, um aumento do vento nos baixos níveis da atmosfera. E concluíram, que existe um efeito combinado de ambos os fatores para o caso estudado.

Estes sistemas convectivos foram classificados por Cohen (1989), em função do seu deslocamento horizontal para o interior da Amazônia em: (i) LI Costeira (LIC), são aquelas cuja propagação horizontal para o interior do continente alcança até $170 \mathrm{~km}$ e não se deslocam continente adentro; (ii) LI com propagação do tipo 1 (LIP1), são as LI com deslocamento horizontal entre 170 e 400 km; e (iii) LI com propagação do tipo 2 (LIP2), são as que apresentam deslocamento horizontal superior a $400 \mathrm{~km}$. As mesmas se desenvolvem frequentemente na costa norte e leste do NEB, com uma extensão horizontal de $1.400 \mathrm{~km}$ de comprimento por $170 \mathrm{~km}$ de largura, ocorrendo maior atividade convectiva no final da tarde ou início da noite (Cavalcanti 1982; Cohen 1989; Garstang et al 1994).

\section{Complexo Convectivo de Mesoescala}

Para a definição do Complexo Convectivo de Mesoescala (CCM) considera-se o tamanho, a forma e o tempo de vida do sistema convectivo, seguindo os critérios de Maddox (1980). (i) Tamanho: o sistema deve apresentar cobertura de nuvens observadas por satélite, com temperaturas no canal infravermelho menores que $-32^{\circ} \mathrm{C}$ numa área de 100.000 $\mathrm{km}^{2}$, sendo que a região mais interna da nuvem deve apresentar temperaturas menores que $-52^{\circ} \mathrm{C}$ na parte interna do SCM com área de $50.000 \mathrm{~km}^{2}$; (ii) Forma: deve ter formato circular com excentricidade maior que 0,7. Estes CCM são associados a uma densa camada de cirrus, e são identificados nas imagens de satélites por ter uma aparência circular (Silva Dias, 1987) e (iii) Tempo de vida: quando as duas características do tamanho ocorrem por um período superior a 6 horas.

Seguindo estes critérios, os CCM são fenômenos que atuam na escala meso- $\alpha$ (de 200 a 2500 km) (Tabela 1), podendo ser considerados agrupamentos de Cumulonimbus, cujas bigornas formam uma cobertura contínua que dá o aspecto típico visto de uma imagem de satélite. Nos casos que as características incluídas na definição de CCM não são verificadas, os sistemas são chamados apenas de SCM. A maior ocorrência de CCM é no período da primavera e verão e a região preferencial é entre as latitudes $15^{\circ}$ e $30^{\circ} \mathrm{S}$. A maioria dos CCM que ocorrem nesta faixa de latitudes tem um ciclo de vida típico, com início de formação à noite ou de madrugada, chegando a sua máxima extensão durante a manhã e dissipando-se por volta do meio dia (Silva Dias et al. 2009). Os CCM da região NEB passam por este mesmo processo sendo de forma tardia. Um CCM provoca chuvas fortes e isoladas de curta duração. Estes são aglomerados de nuvens 
que se formam devido às condições locais favoráveis, tais como: temperatura do ar elevada e menor pressão, assim provocando chuvas fortes com curta duração (Ferreira e Mello 2005). Quando as temperaturas se elevam, as partículas do ar se afastam, ficando menos denso, permitindo que a pressão diminua.

Algumas pesquisas sobre a formação e desenvolvimento dos CCM na AS tem sido abordado por alguns autores, tais como: Guedes 1985; Velasco e Fritsh 1987; Silva Dias 1987; Duquia e Silva Dias 1994; Machado et al 1994; Nieto Ferreira et al 2003; Lima 2004. Este sistema segue quatro etapas para sua formação segundo Maddox (1980): iniciação, desenvolvimento, estágio maduro e dissipação. Na fase de iniciação ocorrem linhas de rajadas, células convectivas isoladas de tempestade em uma região com condições favoráveis à convecção, cada uma com seu próprio ciclo de vida. $\mathrm{Na}$ fase de desenvolvimento, a convergência de ar quente e umidade nos níveis inferiores alimenta o processo de convecção. No estágio maduro os elementos convectivos continuam a sustentar a atividade do sistema, até chegar a dissipação onde a convecção cessa.

Algumas características favorecem o desenvolvimento dos CCM que são: JBN; advecção vertical acentuada; convergência em níveis baixos e divergência em níveis altos; advecção de temperatura quente em baixos níveis e alta umidade no lugar de atuação. Em geral, estes sistemas são continentais e noturnos. De acordo com Velasco e Fritsch (1987), JBN representa um dos mecanismos dinâmicos responsáveis pelo transporte de calor e umidade da Região Amazônica para as regiões de sua formação, alimentando a convecção local.

Uma das condições básicas do CCM é a presença de ar frio e seco com ventos fortes de oeste na média troposfera e advecção de ar quente e úmido na baixa troposfera. Estas condições, em conjunto, geram ocorrência de convecção profunda (Velasco e Fritsch 1987). A maior probabilidade de convecção ocorre quando os ventos formam o maior ângulo possível com as isolinhas de convecção, o que ocasiona um transporte mais rápido de umidade e calor, favorecendo as instabilidades. Em latitudes tropicais, os CCM possuem praticamente o mesmo comportamento das latitudes médias, com exceção da influência dos JBN, e com seu ciclo de vida tendo início mais tardio, e a preferência para sua formação é na região boliviana e colombiana, praticamente durante o ano todo (Velasco e Fritsch 1987; Machado e Laurente 2004; Sakamoto 2009; Sakamoto et al 2011).

Estudos sobre os CCM na AS foram realizados por alguns pesquisadores (Velasco e Fritsch 1987; Machado e Laurente 2004; Sakamoto 2009; Durkee e Mote 2009a e 2009b; Fedorova et al 2009; Sakamoto et al 2011). Velasco e Fritsch (1987) utilizaram critérios semelhantes aos de Maddox (1980) para a região tropical norte e sul (latitudes de $20^{\circ}$ $\mathrm{N}$ e S) e subtropical ao sul de $20^{\circ} \mathrm{S}$, com a diferença apenas nos valores de temperatura, com valores em torno de 233 a $231{ }^{\circ} \mathrm{K}$ ao invés de $241^{\circ} \mathrm{K}$, e 211 a $209^{\circ} \mathrm{K}$ em vez de $221^{\circ} \mathrm{K}$. Como resultado, observaram em ambas as regiões que a formação do sistema ocorre no início da noite (19:00h), ficando mais extenso algumas horas depois (21:30h), com máxima extensão após a meia noite, em torno das 03:00h da madrugada persistindo até as 09:00h da manhã. Também verificaram que sua atuação predomina sobre o nordeste da Argentina, Paraguai e sul do Brasil; estas regiões sãs favorecidas pela influência dos JBN alimentando os mesmos.

Houze (1982) idealizou o ciclo de vida dos SCM tropicais como agrupamentos de torres convectivas com linhas de atuação flutuantes, associados a chuvas fortes. Além disso, a precipitação mais leve se estende por uma distância horizontal de 100-200 km, associado a nuvens estratiforme profunda que se estende da média troposfera até as camadas mais altas, atingindo o escudo de nuvens do tipo cirrus em altos níveis. Posteriormente, Houze (1989) adicionou detalhes a este modelo conceitual, descrevendo aspectos da relação entre as células convectivas profundas e a região estratiforme associada. Seu esquema enfatiza os aspectos microfísicos dos processos de precipitação nas regiões convectivas e estratiformes. Segundo este autor os maiores registros de precipitação ocorrem onde as partículas de neve geradas convectivamente atingem o nível de $0^{\circ} \mathrm{C}$, após a passagem pela nuvem estratiforme (Smull e Houze 1985; Rutledge e Houze 1988). Neste ambiente de movimento vertical, as partículas de neve tendem a precipitar enquanto cresce por deposição de vapor. Na camada entre $0^{\circ} \mathrm{e}-12^{\circ} \mathrm{C}$, as partículas se agregam para formar grandes flocos de neve (Houze e Churchill 1987) e, aparentemente, às vezes crescem também. (Leary e Houze 1979).

Na região NEB os CCM foram analisados por Fedorova et al (2009), no qual concluíram que a influência destes sistemas nos eventos de precipitação intensa do estado de Alagoas, se deve aos que possuem dimensão média de $190.000 \mathrm{~km}^{2}$, com duração de $11 \mathrm{~h}$, tendo seu desenvolvimento máximo pela manhã. 
No NEB os CCM ocorrem com mais frequência nos meses de janeiro e março, ou seja, período de verão (Silveira 2010). Resultado que também, foi encontrado no estudo feito por Albuquerque (2011), no qual, analisou 80 eventos de CCM atuantes no NEB, no período de 10 anos, e identificou que 49\% dos CCM ocorriam no verão e $46 \%$ no outono. Segundo Souza e Alves (1998), os ventos alísios são de fundamental importância no desenvolvimento dos CCM no norte do NEB, por advectar ar quente do oceano para dentro do continente. Como também, a influência do planalto da Borborema, que atua como uma forçante a barlavento empurrando as parcelas de ar para cima tornando-as instáveis (Silveira 2012).

Os parâmetros para localização e intensidade destes sistemas são: Divergência em $200 \mathrm{hPa}$, ômega em 500 hPa, fluxo de umidade, advecção de temperatura em $850 \mathrm{hPa}$, pressão em superfície, vento em 850 hPa, espessura da camada entre 500-100 hPa, CAPE, imagem de satélite e precipitação (EUMeTrain 2012).

Tais sistemas influenciam em todo Brasil, visto que estes SCM podem atuar em conjunto a outro sistema atmosférico. A região com maior frequência é a Bacia do Prata favorecida sua formação pela umidade vinda da Amazônia pelos JBN.

\section{Sistema frontal (SF)}

Os Sistema Frontal (SF) ou frentes (Figura 1) são definidos como uma zona de transição entre duas massas de ar de diferentes densidades e temperaturas, que se aproximam e se mantém individualizadas, conservando suas particularidades (Petterssen 1956; Vianello 1991). Onde elas ocorrem o tempo é instável. Segundo Tubelis e Nascimento (1984), no contato entre duas massas de ar de temperaturas diferentes forma-se uma superfície de descontinuidade, conhecida como superfície frontal. Essa descontinuidade é uma zona de transição, estreita e inclinada, na qual os elementos meteorológicos variam abruptamente. A linha ou zona de contato da superfície frontal com a superfície do solo, ou qualquer outro plano horizontal, é chamada de Frente.

\section{Baixa Troposfera}

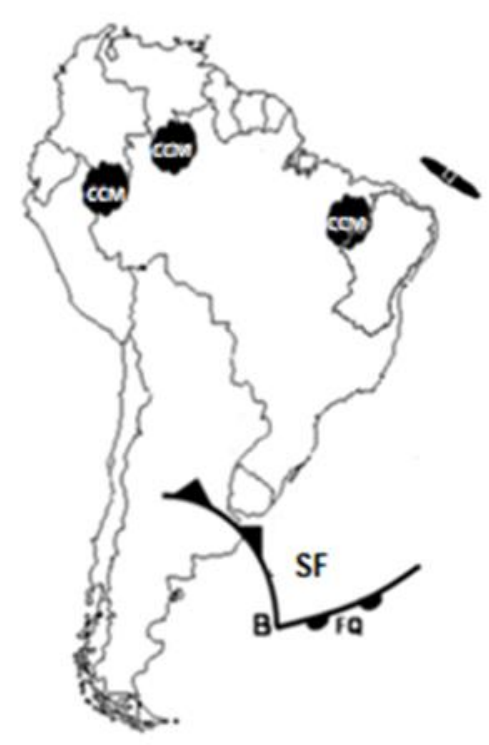

Figura 1 Sistemas meteorológicos atuantes na primeira camada da atmosfera (Baixa Troposfera). Fonte: Adaptado de Reboita (2012).

O termo frente (em analogia as frentes de batalha da Primeira Guerra Mundial) foi proposto por Jacob Bjerknes (1919) no modelo conceitual onde mostrava que durante o processo de convergência dos ventos em superfície, o ar quente, menos denso, sobe sobre o ar frio (cunha de ar frio), este modelo foi chamado de Teoria da Frente Polar. 


\section{Local e período de atuação}

Os SF atuam sobre o continente e Oceano Atlântico adjacente, desde o sul da AS até as latitudes mais baixas, chegando a atingir o NEB e parte oeste da Amazônia (Cavalcanti et al. 2009), onde o efeito principal está ligado à precipitação. Kousky (1979) e Kousky e Ferreira (1981) mostraram que as penetrações dos SF em latitudes baixas, também produzem, um efeito pronunciado na atividade convectiva da faixa tropical da AS.

Segundo Kousky (1979), as frentes frias afetam o clima da AS o ano todo. Em geral, estas frentes apresentam um formato de arco, com orientação NO-SE, sendo facilmente identificadas em imagens de satélite e, geralmente, se deslocam de sudoeste para nordeste sobre o continente e o oceano Atlântico adjacente. Souza (1998) e Molion e Bernardo (2002) explicaram que, durante o outono e inverno, as frentes frias são fontes de pluviosidade para o NEB, principalmente para o setor sul e posteriormente para o leste desta região. A medida que as frentes avançam para as latitudes mais baixas as mesmas enfraquecem, antes de chegar a atingir o leste do NEB. Para que cheguem até as latitudes mais baixas, as frentes frias precisam estar mais intensas. De modo geral, os SF atuam com maior frequência, nas latitudes de $30^{\circ} \mathrm{S}$ e $40^{\circ} \mathrm{S}$ com total de nove eventos por mês; e menor frequência, ao norte de $20^{\circ} \mathrm{S}$, com dois eventos por mês (Satyamurty et al 1998).

\section{Ciclo de vida}

Formação

A formação deste sistema atmosférico ocorre quando há o encontro de duas massas de ar com propriedades distintas. Essas características produzem uma zona ou superfície de descontinuidade que pode ser fria, quando o ar frio avança sobre o ar quente; ou quente, quando o ar quente avança sobre o ar frio. Em ambos os casos, é comum o ar frio que é mais denso e mais pesado empurrar o ar quente para cima, que é mais leve, assim com a ascensão de ar quente e úmido, ocorre a formação de nuvens, gerando instabilidade atmosférica (Kousky 1979).

\section{Desenvolvimento}

As frentes geralmente se formam dentro de um limite baroclínico entre massas de ar frio e quente. O principal processo físico para o desenvolvimento de uma frente fria é o movimento do ar frio contra o ar quente. $\mathrm{O}$ ar quente sobe ao longo do limite enquanto o ar frio desce. Se houver umidade suficiente, o movimento ascendente leva à condensação e ao desenvolvimento de nuvens, consequentemente, a precipitações. A passagem da frente fria geralmente está associada à precipitação, muitas vezes com granizo e trovoadas, com queda acentuada da temperatura, elevação rápida da pressão atmosférica e mudança súbita na direção e intensidade do vento, por sua vez, acompanhada por rajadas, que algumas vezes representam fenômenos adversos. Entretanto, para a passagem da frente quente observa-se que a precipitação é contínua, a temperatura aumenta levemente, a umidade eleva-se rapidamente, a pressão cessa sua diminuição e o vento muda sua direção e velocidade (Petterssen 1956; Ayoade 1996; Torres 2008).

\section{Dissipação}

A atuação dos SF no NEB está reduzida a aproximadamente $20 \%$ das frentes que atuam no Brasil (Kousky 1979). Isso acontece, porque na maioria das vezes, estas se dissipam no meio do caminho. Após sua passagem, normalmente observa-se queda de temperatura, aumento da pressão, rajadas de vento, quando o gradiente de pressão é intenso; a precipitação cessa, ficando o tempo estável, com céu limpo (Petterssen 1956). Após sua passagem seguem-se alguns dias de tempo bom, durante os quais o ar frio vai aos poucos se aquecendo, até que uma outra frente fria realimente o ciclo de chuva/frio/vento/aquecimento/nova frente. Assim, os invernos no Brasil são frios e chuvosos. Devido às influências da massa polar atlântica e de outras massas de ar, o clima subtropical apresenta chuvas durante todo o ano, independentemente da estação (Torres 2008). 
Geralmente, os parâmetros utilizados para localização e intensidade deste tipo de sistema atmosférico são: Divergência em 200 hPa, ômega em 500 hPa, altura geopotencial em 500 hPa, fluxo de umidade, pressão ao nível do mar, advecção de temperatura em $850 \mathrm{hPa}$, advecção de vorticidade em 500 e $300 \mathrm{hPa}$, espessura da camada e vento em 850 hPa (EUMeTrain, 2012)

Os SF influenciam em toda região sul e Sudeste do Brasil, assim como em latitudes mais baixas: sul da Amazônia, centro-oeste e sul do NEB.

\section{Considerações Finais}

Com este artigo foi possível reconhecer as características dos SCM que influenciam na ocorrência de eventos extremos de chuva no SANEB, permitindo a tomada de decisão antecipada, minimizando os prejuízos tanto sócio econômico como ambiental.

\section{Referências}

Albuquerque CSM (2011) Desenvolvimento e trajetórias dos complexos convectivos de Mesoescala no estado de Alagoas entre os anos de 1999 e 2009. Trabalho de conclusão de curso, ICAT/UFAL.

Ayoade JO (1996) Introdução à climatologia para os trópicos. DIFEL, São Paulo.

Barbosa TFB, Correia MF (2005) Sistemas convectivos intensos no Semiárido Brasileiro: O controle da grande escala. Revista Brasileira de Meteorologia 20:395-410.

Bjerknes J (1919) On the Structure of Moving Cyclones. Monthly Weather Review 47:95-99.

Carvalho LMV, Jones C (2009) Zona de Convergência do Atlântico Sul. In: Tempo e Clima no Brasil. Cavalcanti IFA, Ferreira NJ, Silva MGAJ, Dias, MAFS (org.). São Paulo: Oficina de Textos, pp. 95-109.

Cavalcanti I, Ferreira N, Silva M, Silva-Dias M (2009) Tempo e Clima no Brasil, Oficina de Textos.

Cavalcanti IFA (1982) Um estudo sobre interações entre sistemas de circulação de escala sinótica e circulações locais. Dissertação Mestrado em Meteorologia), INPE, São José dos Campos.

Cohen JCP, Dias MAFS, Nobre CA (1995) Enviromental conditions associated with amazonian squall lines: A case study. Monthly Weather Review 123:3163-3174.

Cohen JCP, Dias MAFS, Nobre CA (1989) Aspectos climatológicos das linhas de instabilidade na Amazônia. Climanálise - Boletim de Monitoramento e Análise Climática 4:34-40.

Cohen JCP, Cavalcanti IFA, Braga RHM (2009) Linhas de instabilidade na costa N-NE da América do Sul. In. Cavalcanti IFA, Ferreira NJ, Silva MGAJ, Dias MAFS. (Orgs.). Tempo e clima no Brasil. São Paulo: Oficina de Texto, pp. 75-92.

Costa MS, Lima KC, Gonçalves WA, Mattos EV (2019) Sistemas Convectivos de Mesosescala Associados a Eventos Extremos de Precipitação Sobre o Semiárido do Nordeste do Brasil. Anuário do Instituto de Geociências - UFRJ 42:317-328

Cotton WR, Lin MS, Mcanelly RL, Tremback CJ (1989) A composite model of mesoscale convective complexes. Monthly Weather Review 117:765- 783

Duquia CG, Silva-Dias MAF (1994) Complexo convectivo de mesoescala: um estudo de caso para o oeste do Rio Grande do Sul. In: Congresso Brasileiro De Meteorologia, Anais, Belo Horizonte, SBMET, pp. 610-612.

Durkee J, Mote T, Shepered JM (2009a) The Contribution of Mesoscale Convective Complexes to Rainfall across Subtropical South America. International Journal of Climatology.

Durkee JD, Mote TL (2009b) A climatology of warm-season mesoscale convective complexes in subtropical South America. International Journal of Climatology. doi:10.1002/joc.1961.

Eichholz CW (2011) Características físicas dos Sistemas Convectivos de Mesoescala que afetaram o Rio Grande do Sul no período de 2004 a 2008. Programa de Pós-Graduação em Meteorologia, Universidade Federal de Pelotas. Dissertação de Mestrado, 102p.

Eichholz CW, Campos CRJ (2009) Características Físicas dos Sistemas Convectivos de Mesoescala que afetaram o Rio Grande do Sul em 2006. Anuário do Instituto de Geociências - UFRJ 37:70-80. 
Fedorova N, Levit V, Rodrigues LRL, Costa SB (2009) Mesoescale Convective complex on the Northeastern Coast of Brazil. Journal of the Georgian Geophysical Society 13:36-49.

Ferreira AG, Mello NGS (2005) Principais Sistemas Atmosféricos Atuantes sobre a Região Nordeste do Brasil e a Influência dos Oceanos Pacífico e Atlântico no Clima da Região. Revista Brasileira de Climatologia 1:15-26.

Garstang M, Massie Jr HL, Halverson J, Greco S, Scala J (1994) Amazon Coastal Squall Lines. Part I: Structure and Kinematics. Monthly Weather Review 122:608-622.

Guedes RL (1985) Condições de grande escala associadas a sistemas convectivos de mesoescala sobre a região central da América do Sul. Dissertação de Mestrado, Dep. Meteorologia, IAG-USP.

Guedes RL, Machado LAT, Silveira JMB, Alves MAS, Waltz RC (1994) Trajetórias dos sistemas convectivos sobre o continente americano. In: Congresso Brasileiro De Meteorologia, 8, Belo Horizonte, Anais, Belo Horizonte, SBMET, pp. 77-80.

Houze RAJr (1977) Structure and dynamics of a tropical squall line system. Monthly Weather Review 105:1540-1567.

Houze RAJr (1982) Cloud clusters and large-scale vertical motions in the tropics. J Meteor Soc Japan 60:396-410.

Houze RAJr (1989) Observed structure of mesoscale convective systems and implications for large-scale heating. Quart J Roy Meteor Soc 115:425-461.

Houze RAJr (2004) Mesoscale convective systems. Rev Geophys. doi:10.1029/2004RG000150.

Houze RAJr, Smull BF, Dodge P (1990) Mesoscale organization of springtime rainstorms in Oklahoma. Monthly Weather Review 118:613-654.

Houze RA (1993) Mesoscale convective systems. In: Houze RA (ed.). Cloud dynamics. Academic Press, Inc., 53:334-404.

Kousky VE (1979) Frontal influences on Northeast Brazil. Monthly Weather Review 1:1140-1153.

Kousky VE (1980) Diurnal rainfall variation in Northeast Brazil. Monthly Weather Review 108:488-498.

Kousky VE, Ferreira NJ (1981) Interdiurnal surface pressure variation in Brazil: Their spatial distribution, origins and effects. Monthly Weather Review 109:1999-2008.

Laing AG, Fritsch JM (2000) The large-scale environments of the global populations of mesoscale convective complexes Monthly Weather Review, 128: 2756-2776.

Leary CA, Houze Jr RA (1979) The structure and evolution of convection in a tropical cloud cluster. J Atmos Sci 36:437-457.

Lima ECS (2004) Análise de um Complexo Convectivo de Mesoescala (CCM) observado na região norte da Argentina no dia 27 de novembro de 2002: Um estudo de caso utilizando o modelo RAMS. Dissertação de Mestrado, Programa de Pós-graduação em Meteorologia, Universidade Federal de Pelotas.

Macedo SR, Vila D, Machado LA (2004) Fortracc - Previsão a curto prazo e evolução dos sistemas convectivos: FORTRACC V1.1 - Guia de Usuário, São Paulo. INPE/ CPTEC, Disponível em <http://sigma.cptec.inpe.br/fortracc/pdf/fortracc.pdf>.

Machado LAT, Laurent H (2004) The convective system area expansion over Amazonia and its relationships with convective system life duration and high-level wind divergence. Monthly Weather Review 132:714-725

Machado LAT, Rossow WB (1993) Structural characteristics and radiative of tropical cloud clusters. Monthly Weather Review 121:3234-3260.

Machado LAT, Guedes RL, Silveira JMB, Waltz RC, Alves MAS (1994) Ciclo de vida de sistemas convectivos. In: Congresso Brasileiro De Meteorologia, 8, Belo Horizonte, 1994. Anais, Belo Horizonte, SBMet, pp. 323- 326.

Maddox RA (1983) Large-scale meteorological conditions associated with midlatitude, mesoscale convective complexes. Monthly Weather Review 111:1475-1493

Maddox RA (1980) An objective technique for separating macroscale and mesoscale features in Meteorological data. Monthly Weather Review 108:1108-1121.

Maddox RA (1980) Mesoscale convective complexes. Bulletin American Meteorological Society 61:1374-1387.

Mcanelly RL, Cotton WR (1989) The Precipitation Life Cycle of Mesoscale Convective Complexes over the Central United States. Monthly Weather Review 117:784-808.

Molion LCB, Bernardo SO (2002) Uma revisão da dinâmica das chuvas no nordeste brasileiro. Revista Brasileira de Meteorologia 17:110.

Nieto Ferreira R, Rickenback TM, Herdies DL, Carvalho LMV (2003) Variability of South American convective clouds systems and tropospheric circulation during januarymarch 1998 and 1999. Monthly Weather Review 131:961-973. 
Orlanski I (1975) A rational subdivision of scales for atmospheric processes. Bulletin of the American Meteorological Society 56:527530

Petterssen S (1956) Weather Analysis and Forecasting. New York, Mc Graw-Hill.

Petterssen S (1956) Motion and Motion systems. In: Weat. Forecasting. New York, Toronto, London: McGraw-Hill.

Pinto LB, Campos CRJ (2007) Ambiente sinótico e perfis verticais de um Sistema Convectivo de Mesoescala (SCM). Revista Brasileira de Geofísica 25:31-43.

Ramos RPL (1975) Precipitation characteristic in the Northeast Brazil dry region. Journal of Geophysical Research 80:1665-1678.

Rutledge AS, Houze RA, Biggerstaff Jr MI, Matejka T, (1988) The Oklahoma-Kansas mesoscale convective system of 10-11 June 1985: Precipitation structure and single-Doppler radar analysis. Monthly Weather Review 116:1409-1430.

Sakamoto MS, Ambrizzi T, Poveda G. (2011) Moisture Sources and Life Cycle of Convective Systems over Western Colombia. Hindawi Publishing Corporation Advances in Meteorology 41:890759.

Sakamoto MS (2009) Sistemas Convectivos de Mesoescala observados na Região Subtropical da América do Sul durante o SALLEX. Programa de Pós-graduação em Meteorologia, Universidade de São Paulo, Tese de Doutorado, 243p.

Salio P, Nicolini M, Zipser EJ (2007) Mesoscale Convective Systems over Southeastern South America and Their Relationship with the South American Low-Level Jet. Monthly Weather Review, 135:1290-1309.

Satyamurti P, Nobre CD, Dias PLS (1998) South America. Meteorology of the Southern Hemisphere, DJ Karoly and DG Vincent, Eds., Amer Meteoro Soc. pp. 119-139.

Silva Dias MAF (1987) Sistemas de mesoescala e previsão de tempo a curto prazo. Revista Brasileira de Meteorologia 2:133-150.

Silva Dias MAF, Rozante JR, Machado LAT (2009) Complexos Convectivos de Mesoescala na América do Sul. In: Cavalcanti IFA. Tempo e clima no Brasil. São Paulo, Oficina de Textos. pp. 181-194

Silveira MHF (2010) Complexos Convectivos de Mesoescala no estado de Alagoas. Trabalho de conclusão de curso em Meteorologia, ICAT/UFAL, 65p.

Silveira MHS (2012) Análise termodinâmica dos complexos convectivos de mesoescala atuantes na costa leste do nordeste brasileiro. Dissertação de mestrado em meteorologia, ICAT/UFAL. 59p.

Smull BF, Houze Jr RA (1985) A midlatitude squall line with a trailing region of stratiform rain, 1985: Radar and satellite observations. Monthly Weather Review 113:117-133.

Song F, Feng Z, Leung LR (2019) Contrasting spring and summer large-scale environments associated with mesoscale convective systems over the U.S. Great Plains. J Clim. doi: 10.1175/JCLI-D-18-0839.1.

Souza EB, Alves JMB, Repelli CA (1998) Um Complexo Convectivo de Mesoescala Associado à Precipitação Intensa sobre FortalezaCE. Revista Brasileira de Meteorologia 13:1-14.

Torres FTP (2008) Introdução à climatologia. Ubá, MG: Geographica.

Torres JC (2003) Sistemas convectivos en mesoescala altamente precipitantes en El norte y centro de Argentina. Programa de Pósgraduação em Ciências. Faculdade de Ciências Exatas e Naturais, Universidade de Buenos Aires, Tese de doutorado, 130p.

Tubelis A, Nascimento FCL (1984) Meteorologia descritiva. Fundamentos e aplicações brasileiras. São Paulo, Nobel, 374p.

Velasco I, Fritsch JM (1987) Mesoscale convective complexes in the Americas. Journal Geophysical Research 92:9591-9617.

Vianello RL, Alves AR (1991) Meteorologia Básica e Aplicações, Universidade Federal de Viçosa, Minas Gerais, MG. 